\title{
Two-stage hepatectomy for liver metastasis from colorectal cancer
}

\author{
Keegan Selby BHSc, Roberto Hernandez-Alejandro MD
}

$\mathrm{C}$ olorectal cancer is the third most common type of cancer in Western countries. In 2012 alone, an estimated 23300 Canadians received a diagnosis of colorectal cancer and 9200 died from it. ${ }^{1}$ Of the patients who receive a diagnosis, about one-third will have metastasis confined to the liver, ${ }^{2}$ but only $10 \%-25 \%$ of these patients will be surgical candidates based on the extent of their disease. ${ }^{3}$ The remainder of patients with metastasis confined to the liver are not considered surgical candidates because removal of the extensive tumour load would result in a very small liver remnant, with ensuing liver failure, hepatic encephalopathy and eventual death. Yet, patients with advanced liver tumours from metastatic colorectal cancer who would previously have been considered for palliative care may now have an option for surgical treatment intended to cure.

Traditional treatment for extensive tumour load of the liver has been neoadjuvant chemotherapy, followed by two stages of surgery. The first stage of surgery involves occlusion of the portal vein feeding the diseased segment of the liver by either embolization or ligation. This induces hypertrophy of the future contralateral healthy

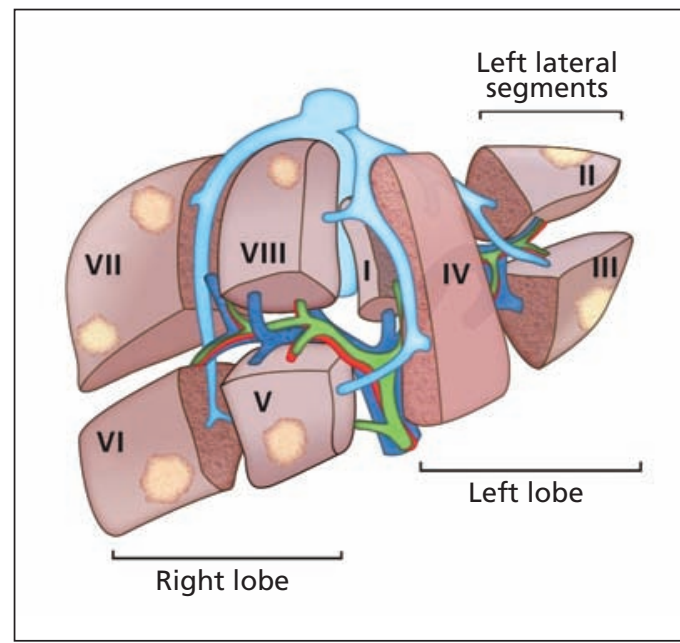

Figure 1: The eight segments of the liver. Only segments I and IV are tumour free in this diagram. liver remnant supplied by the remaining portal vein, so that the second stage (hepatectomy of the diseased liver) does not result in complete liver failure. In a liver exposed to chemotherapy, a future liver remnant of at least $30 \%$ of total liver volume is generally considered necessary to prevent postoperative liver failure. ${ }^{4} \mathrm{~A}$ study by Shindoh and colleagues ${ }^{4}$ involving 194 patients showed a $10 \%$ rate of postoperative liver failure when this target was achieved. Unfortunately, this traditional technique requires about two months to generate the required hypertrophy. During this time, one-third of patients will either have further tumour progression or will not have sufficient hypertrophy of the healthy liver. ${ }^{5}$

Recently, an innovative procedure known as an associating liver partition with portal vein ligation for staged hepatectomy (ALPPS) has become available in Canada. The goal of this procedure is to remove all liver disease within one week and prevent postoperative liver failure.

\section{What is the procedure?}

The ALPPS procedure is similar to traditional treatment in that it is a two-staged procedure for patients requiring extensive hepatectomy that would result in a future liver remnant less than $30 \%$ of total liver volume. ${ }^{6}$ Stage 1 involves sur-
Competing interests: None declared.

This article has been peer reviewed.

The authors have obtained patient consent.

Correspondence to:

Roberto HernandezAlejandro;

roberto.hernandezalejandro @lhsc.on.ca

CMAJ 2014. DOI: 10.1503 /cmaj.131022

\section{KEY POINTS}

- Extensive liver metastasis limits options for surgical treatment.

- A procedure known as associating liver partition with portal vein ligation for staged hepatectomy (ALPPS) increases surgical candidacy, lowers risk of postoperative liver failure and decreases the risk of disease progression.

- Further study is required before the technique can be widely recommended. 
gical removal of any diseased tissue in the left lateral segments of the liver (Figure 1). Next, the right portal vein is selectively ligated, followed

\section{Box 1: Case description \\ To illustrate the procedure known as associating liver partition with portal vein ligation for staged hepatectomy (ALPPS), we describe a case in which we successfully completed this innovative technique. Our patient had sigmoid adenocarcinoma and advanced liver metastasis, deemed unresectable by three academic centres. The only liver segments free of tumour were segments I and IV (Figure 1).}

The patient underwent six cycles of chemotherapy for downsizing of the tumour load. Computed tomography (CT) (Figure 2A) identified the volume of tumour-free liver as only $16 \%$ of total liver volume, which would be insufficient as a remnant.

The primary tumour showed a good response to chemotherapy and, because there was no evidence of bowel obstruction or bleeding, we decided to remove the liver metastasis first, followed by resection of the primary colon tumour at a later date. A modified ALPPS procedure was performed with the aim of preserving the tumour-free segments I and IV. In stage 1, we completely resected the left lateral segments (II and III), and ligated the right portal vein. We then partitioned the liver along the Cantlie line, between the diseased right lobe and the remaining segment IV, to complete stage 1 (Figure 3).

Seven days after the first stage, CT showed hypertrophy of $93 \%$ of the healthy remnant, which nearly doubled in size to $31 \%$ of total liver volume (Figure 2B).

Stage 2 of the ALPPS procedure involved completion of the right hepatectomy (Figure 4). The surgery was successful, although transient cholestasis developed (bilirubin $<120 \mathrm{mmol} / \mathrm{L}$ ), which resolved in four weeks with good liver synthetic function (international normalized ratio 1.2-1.4). The colorectal surgery was completed seven weeks later with no complications, allowing quick return to chemotherapy. At 18 months, there was no evidence of cancer recurrence. by surgical partition of the liver through its parenchyma. The right deportalized hemiliver is now only supplied by its hepatic artery and drained by its respective hepatic veins. This allows it to behave as an auxiliary liver, thus maintaining liver synthetic function and avoiding liver failure during the one-week interval before it is removed. We believe that the liver partition in ALPPS is responsible for the rapid hypertrophy, because, in contrast to traditional treatment, it prevents any collateral portal blood flow between the two sides of the liver. The future liver remnant thus receives $100 \%$ of portal inflow, stimulating the rapid hypertrophy that we observe within one week. In the second stage, the patient returns to surgery to have the tumourfilled, deportalized hemiliver removed, without subsequent postoperative liver failure. ${ }^{6}$ Box 1 describes a case in which this procedure was used. A video describing this procedure is available at www.youtube.com/watch?v=VQuegI _wGhk.

The technique prevents tumour progression and reduces the risk of postoperative liver failure, while allowing return to potential adjuvant chemotherapy sooner. $^{6}$

\section{Who is eligible?}

In patients with primary or secondary liver tumours that are initially unresectable because of a future liver remnant of less than $30 \%$ of total liver volume, an ALPPS procedure may be considered after discussion at a multidisciplinary
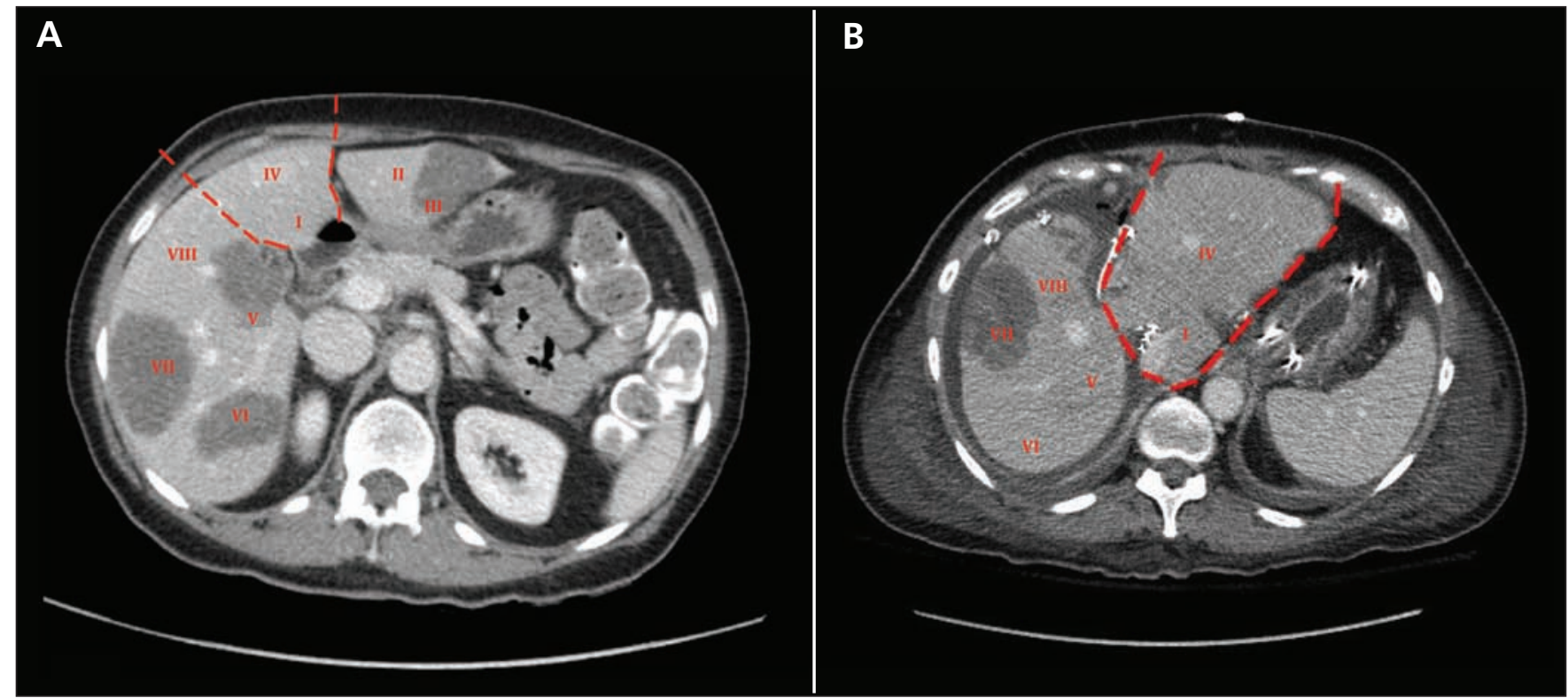

Figure 2: (A) Computed tomographic (CT) image of the abdomen showing widespread liver metastasis in a patient with a primary adenocarcinoma of the sigmoid colon. The only disease-free segments are I and IV (outlined in red). This will become the future liver remnant. (B) One week after stage 1, CT imaging showed substantial hypertrophy of the future liver remnant (outlined in red), which nearly doubled in size to $31 \%$ of total liver volume. 
tumour board. ${ }^{7.8}$ These patients must be deemed medically fit by an anesthesiologist and internal medicine specialist to withstand the back-to-back major abdominal surgeries involved. Previous chemotherapy is not a contraindication; however, caution should be taken if the patient has received more than eight cycles (i.e., because of increased risk of liver toxicity). Because this surgery is intended to cure, patients with major extrahepatic disease that is not amenable to treatment would not be considered candidates. ${ }^{7,8}$

\section{What are the possible harms?}

The complications associated with an ALPPS procedure mimic those of a traditional extended surgical resection, but it is believed that the chances of postoperative liver failure are lower. A case series by Schnitzbauer and colleagues ${ }^{7}$ involving 25 patients reported $12 \%$ mortality, with all deaths occurring after the second phase of this complex procedure. This study included patients who had a variety of primary tumours, including cholangiocarcinomas and ovarian cancer, which may have affected the outcomes. Furthermore, as with any new surgical technique, there is a steep learning curve. It is expected that with surgeon experience, complications will decrease. We believe the strongest factors associated with poor outcomes are patient selection and surgical technique. ${ }^{?}$

There is an ongoing case series involving 15 patients with liver metastasis from colorectal cancer at our centre. It is too early to analyze long-term oncological results; however, our early results show $100 \%$ survival with $13 \%$ recurrence at a median follow-up of nine months. We have experienced a major morbidity rate of $30 \%$, which includes one abdominal wall dehiscence, two intra-abdominal abscesses requiring percutaneous drainage and three cases of prolonged nonobstructive jaundice that resolved within six weeks.

Finally, traditional approaches require a percutaneous embolization of the portal vein, as well as one laparotomy. The ALPPS procedure requires two separate laparotomies, which further increases risks associated with major surgery and general anesthesia. ${ }^{9}$

\section{What is the evidence so far?}

The ALPPS technique has been shown to induce hypertrophy of the future liver remnant that is more robust than with other techniques. Authors have reported a $74 \%$ volume increase of the remnant in a mean of nine days. ${ }^{7}$ The ALPPS pro cedure has also been compared with the wellestablished technique of portal vein embolization/

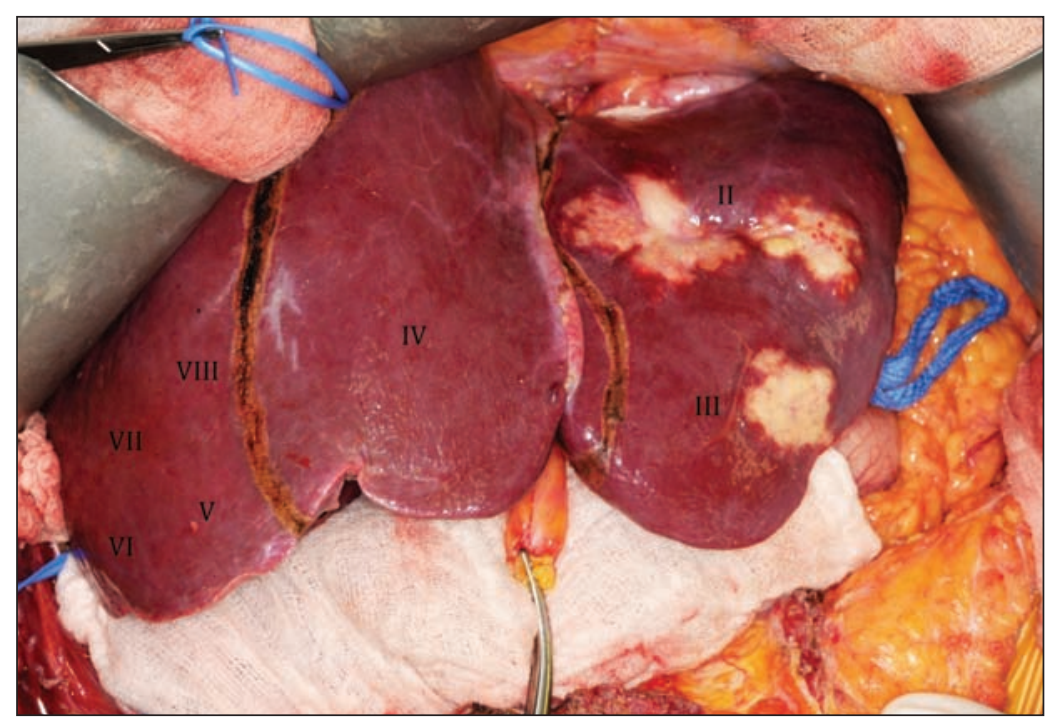

Figure 3: Operative image during stage 1 showing (from the reader's left to right) the right lobe of the liver (segments V-VIII), which will remain partitioned in situ until its removal during stage 2; the healthy future liver remnant (segment IV); and the diseased left lateral segments (II and III), which will be removed during completion of stage 1 .

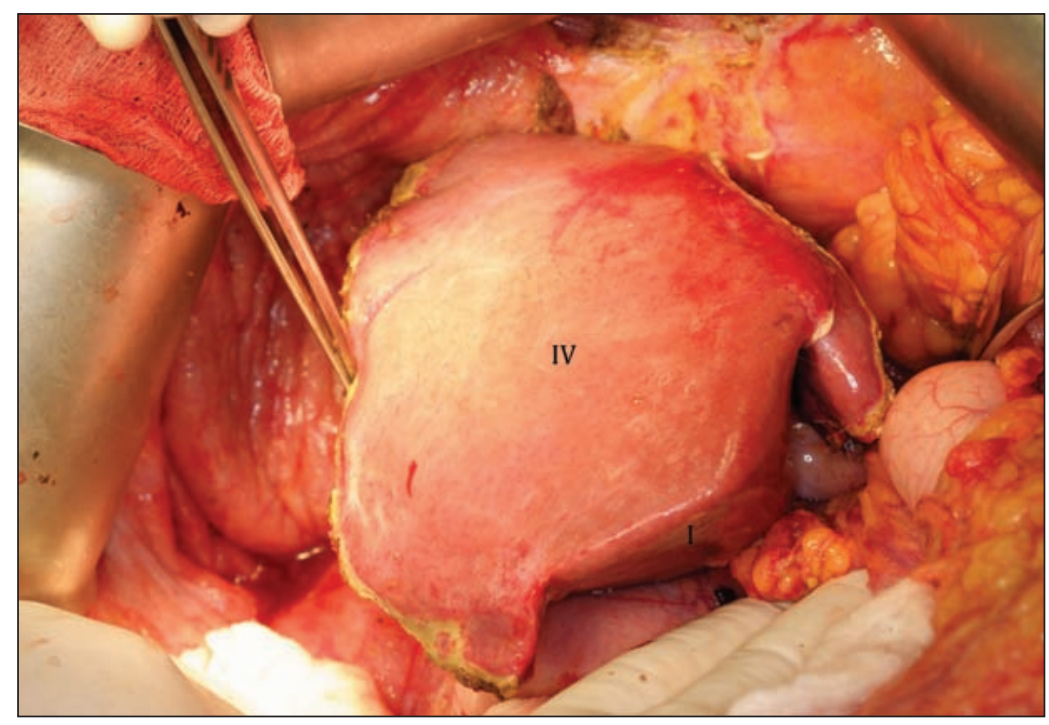

Figure 4: Operative image of the healthy, hypertrophied liver remnant after completion of stage 2 .

ligation (PVE/PVL)..$^{10}$ These comparisons have shown that $79 \%$ of patients undergoing ALPPS achieved cancer-free resection at three months compared with $58 \%$ in the PVE/PVL arm (odds ratio 2.80 , confidence interval $1.12-6.98, p=$ $0.027)$. Volume increases per day were 11 times more rapid with ALPPS (34.8, interquartile range [IQR] 26.4-48.5, cc/d) than with PVE/PVL (2.8, IQR 1.7-5.8, cc/d; $p=0.001)$. Mortality after nine days was $14.6 \%$ with ALPPS and 6.0\% with PVE/PVL $(p=0.20) .{ }^{10}$ Because the procedure is relatively new, most publications have been case series or retrospective comparisons. 


\section{What can we expect in the future?}

Because of the technical complexity of ALPPS surgery, it is likely that this technique will continue to be done only in specialized centres with adequate expertise. Nevertheless, the technique is being used worldwide, with more than 200 cases registered with the ALPPS registry (www.alpps.net/?q = registry).

A recent review by Donati and colleagues ${ }^{11}$ summarized the available ALPPS case series, and concluded that multicentre randomized controlled trials with longer follow-up attempting to delineate the role of ALPPS in liver surgery are required. In Canada, the London Health Sciences Centre is participating in research studies with this goal in mind.

\section{References}

1. Canadian cancer statistics 2012. Toronto (ON): Canadian Cancer Society; 2012. Available: www.cancer.ca/en/about-us/for-media /media-releases/new-brunswick/2012/canadian-cancer-statistics -2012/?region=nb (accessed 2013 May 28).

2. Clavien PA. Malignant liver tumors: current and emerging therapies. Chichester (UK): Wiley-Blackwell; 2010.

3. Kemeny N. Presurgical chemotherapy in patients being considered for liver resection. Oncologist 2007;12:825-39.
4. Shindoh J, Tzeng C, Aloia T, et al. Optimal future liver remnant in patients treated with extensive preoperative chemotherapy for colorectal liver metastases. Ann Surg Oncol 2013;20:2493-500.

5. Wicherts DA, Miller R, de Haas RJ, et al. Long-term results of two-stage hepatectomy for irresectable colorectal cancer liver metastases. Ann Surg 2008;248:994-1005.

6. Torres OJM, Moraes-Junior JMA, Lima NCL. Associating liver partition and portal vein ligation for staged hepatectomy (ALPPS): a new approach in liver resections. Arq Bras Cir Dig 2012;25:290-2.

7. Schnitzbauer AA, Lang SA, Goessmann H, et al. Right portal vein ligation combined with in situ splitting induces rapid left lateral liver lobe hypertrophy enabling two-staged extended right hepatic resection in small-for-size settings. Ann Surg 2012;255:405-14.

8. Knoefel WT, Gabor I, Rehders A, et al. In situ liver transection with portal vein ligation for rapid growth of the future liver remnant in two-stage liver resection. Br J Surg 2013;100:388-94.

9. Jain HA, Bharathy KG, Negi SS. Associating liver partition and portal vein ligation for staged hepatectomy: Will the morbidity of an additional surgery be outweighed by better patient outcomes in the long-term? Ann Surg 2012;256:e10.

10. Schadde E, Ardiles V, Slankamenac K, et al. ALPPS offers a better chance of complete resection in patients with primarily unresectable liver tumors compared with conventional-staged hepatectomies: results of a multicenter analysis. World J Surg 2014;38: 1510-9.

11. Donati M, Stavrou GA, Oldhafer KJ. Current position of ALPPS in the surgical landscape of CRLM treatment proposals. World $J$ Gastroenterol 2013;19:6548-54

Affiliations: Schulich School of Medicine and Dentistry (Selby), Western University; London Health Sciences Centre (Hernandez-Alejandro), Western University, London, Ont.

Contributors: Both authors drafted and revised the article, and approved the final version submitted for publication. 